\title{
Telomere biology is differently affected within clinical subsets of systemic sclerosis and points towards different downstream defects
}

\author{
Jasper CA Broen ${ }^{1 *}$, Liane McGlynn², Meng May Chee ${ }^{2}$, Lenny Geurts-van Bon ${ }^{1}$, Robert L Lafyatis ${ }^{3}$, \\ Timothy RDJ Radstake ${ }^{1,3}$, Paul G Shiels ${ }^{2}$ \\ From 7th European Workshop on Immune-Mediated Inflammatory Diseases \\ Noordwijk aan Zee, the Netherlands. 28-30 November 2012
}

\section{Background}

Systemic sclerosis is a immune mediated inflammatory disease culminating in vasculopathy and extensive fibrosis of the skin and internal organs. Telomere shortening has previously been described in SSc.

\begin{abstract}
Aim
To replicate previous findings in large cohort, investigate telomere shortening in multiple immune celltypes and scrutinize underlying aberrances in telosome gene expression.
\end{abstract}

\section{Methods}

We measured telomere length by PCR in a cohort of 185 SSc patients and 100 healthy controls. Next we investigated plasmacytoid dendritic cells, $\mathrm{T}$ cells, B cells, monocytes and myeloid dendritic cells from 25 SSc patients for cell specific telomere attrition. Finally we investigated whether there were differences in expression of 31 genes involved in telomere pathways.

\section{Results}

We observed a significant age related telomere attrition in healthy controls and lcSSc patients (Both $p<0.001$ ), but not in dcSSc patients. In the immune cell subset specific analysis we observed significant shorter telomeres in $B$ cells and myeloid dendritic cells of both lcSSc and $\mathrm{dcSSc}$ patients (B-Cells $p=0.014, p=0.002 \&$ myDCs $p=0.019, p=0.004$ respectively). PDCs and T cells were significantly shorter in dcSSc patients only $(\mathrm{p}=0.001$ and $\mathrm{p}=0.003$ respectively). In addition, we observed in early
SSc, that B cells exhibit a significant upregulation of the telosome genes SIRT6, RIF1 and WRN (after correction for multiple testing $\mathrm{p}=0.03,0.006$ and 0.048 respectively). In later disease there is a significant higher expression of HDAC9 in monocytes from dcSSc compared to lcSSc patients. Intriguingly, in PDCs of diffuse SSc patients, regardless whether it is early or progressed disease the expression of SIRT1 is significantly lower ( $\mathrm{p}=0.002$ in all comparisons).

\section{Conclusions}

Aberrances in telomere shortening and biology are a feature of SSc, reflecting a difference in clinical subsets at the cellular level.

\section{Author details}

${ }^{1}$ Dept. of Rheumatology and Clinical Immunology, University Utrecht Medical Center, Utrecht, the Netherlands. ${ }^{2}$ Dept. of Cancer Sciences, University of Glasgow, Glasgow, Scotland, UK. ${ }^{3}$ Dept. of Rheumatology, Boston University Medical Center, Boston, MA, USA.

Published: 28 November 2012

\section{doi:10.1186/1479-5876-10-S3-P37}

Cite this article as: Broen et al:: Telomere biology is differently affected within clinical subsets of systemic sclerosis and points towards different downstream defects. Journal of Translational Medicine 2012 10(Suppl 3): P37. 